\title{
Assessment of Bidirectional Relationships Between 98 Genera of the Human Gut Microbiota and Amyotrophic Lateral Sclerosis: A 2-Sample Mendelian Randomization Study
}

\author{
Linjing Zhang \\ Peking University Third Hospital \\ Zhenhuang Zhuang \\ Peking University \\ Gan Zhang \\ Peking University Third Hospital \\ Tao Huang \\ Peking University
}

Dongsheng Fan ( $\sim$ dsfan@sina.com )

Peking University Third Hospital https://orcid.org/0000-0002-7080-3971

\section{Research}

Keywords: Amyotrophic lateral sclerosis, gut microbiota, gamma-glutamyl amino acids, bidirectional relationships, two-sample Mendelian randomization

Posted Date: May 24th, 2021

DOl: https://doi.org/10.21203/rs.3.rs-526836/v1

License: (c) (i) This work is licensed under a Creative Commons Attribution 4.0 International License.

Read Full License

Version of Record: A version of this preprint was published at BMC Neurology on January 3rd, 2022. See the published version at https://doi.org/10.1186/s12883-021-02522-z. 


\section{Abstract}

\section{Background}

Growing evidence suggests a mutual interaction between gut microbiome alterations and ALS pathogenesis. However, previous studies were susceptible to potential confounding factors and reverse causation bias, likely leading to inconsistent and biased results. Therefore, to decipher the potentially mutual relationship between gut microbiota and ALS, we used a bidirectional two-sample MR approach to examine the associations between the gut microbiome and ALS. Effects of potential metabolites on ALS were also estimated in MR design.

\section{Results}

Using the inverse variance-weighted method, OTU10032 unclassified Enterobacteriaceae species-level OTU and unclassified Acidaminococcaceae were associated with a higher risk of ALS (per relative abundance: OR, 1.04; $95 \% \mathrm{Cl}, 1.01-1.07 ; P=0.011$ and $\mathrm{OR}, 1.02 ; 95 \% \mathrm{Cl}, 1.01-1.04 ; P=0.009$, respectively). Importantly, Gamma-Glu-Phe was showed potential deleterious effects on the risk of ALS (genetically predicted per a 1-standard deviation increase in the level of Gamma-Glu-Phe: OR, 1.96; 95\% $\mathrm{Cl}, 1.50-2.55 ; P=0.012)$. Sensitivity analysis of the two candidate genera and metabolites using the MREgger and weighted-median methods produced similar estimates, and no horizontal pleiotropy or outliers were observed. Intriguingly, genetically predicted ALS was associated with an increase in the relative abundance of OTU4607_Sutterella (per 1-unit higher log odds: $\beta, 2.23 ; 95 \% \mathrm{Cl}, 1.27-3.18 ; P=0.020$ ) and Lactobacillales_ORDER (per 1-unit higher log odds: $\beta, 0.51 ; 95 \% \mathrm{Cl}, 0.09-0.94 ; P=0.019$ ).

\section{Conclusions}

Our findings provide novel evidence supporting the bidirectional relationship between the gut microbiota and ALS and highlight that a transsynaptic, glutaminergic, excitotoxic mechanism could provide a pathogenic basis for ALS. These results may contribute to designing microbiome- and microbiomedependent metabolite interventions in future ALS clinical trials.

\section{Background}

Amyotrophic lateral sclerosis (ALS) is a fatal neurodegenerative motor neuron disease accompanied by both systemic and central nervous system-specific inflammation as well as energy dysmetabolism [1-3]. These potential pathogenetic factors have recently been found to mutually interact with the gut microbiota $[4,5]$, suggesting that the gut microbiota could be involved in the development of the disease and be affected by the disease vice versa.

Observational studies have shown that the interface between the host and the gut microbiome may be altered in mouse models of ALS [6, 7], including impaired gut barrier function and a dysbiotic microbiome configuration that can be partially corrected by butyrate supplementation [7]. Studies of whether gut 
microbiome dysbiosis occurs between ALS patients and healthy controls have yielded conflicting results [8-10]. Notably, a recent study [11] of 11 distinct commensal bacteria based on their individual supplementation into antibiotic-treated Sod1-Tg mice found that Akkermansia muciniphila (AM) and AMassociated nicotinamide ameliorate symptoms of ALS. In humans, distinct microbiome and metabolite configurations have been observed in a small preliminary study that compared 37 patients with ALS with household controls [11].

Growing but conflicting evidence is attractive, raising the hypothesis of a mutual interaction between gut microbiome alterations and ALS pathogenesis. However, it has been difficult to determine whether these changes in the intestinal microbiota are causative of ALS disease, an exacerbating factor for disease, or a consequence of disease. The composition and diversity of the gut microbiome can be easily altered as a result of bacterial infections, antibiotic treatment, lifestyle changes, surgery, and long-term changes in diet. Available evidence is in large part inadequate, as observational studies are susceptible to these potential confounding and reverse causation biases, which can lead to inconsistent and biased results. To some extent, data from antibiotic-treated Sod1-Tg mice could demonstrate causal relationships but are scarce, and the number of commensal bacteria that have been investigated is limited.

The Mendelian randomization (MR) approach is a widely used genetic epidemiological method for assessing causal associations between risk factors and disease by exploiting genetic variants as instrumental variables (IVs) for exposure [12-14]. This approach is less likely to be affected by the confounding or reverse causation bias that exists in observational findings.

Therefore, to decipher the potentially mutual relationship between the gut microbiota and ALS, we used a bidirectional two-sample MR approach to examine the associations between the gut microbiome and ALS (Figure 1). Notably, the gut microbiome is remote from the disease site of ALS, it is suggested that a potential systemic influx of microbiome-regulated metabolites may affect the susceptibility of motor neurons in ALS. We also estimated the effects of potential metabolites on ALS in MR design.

\section{Methods}

The detailed approach of selection of IVs for exposures, genome-wide association study (GWAS) summary statistics for ALS, and MR analysis were previously described [15]. The MR approach we used was based on the following three assumptions: 1) genetic variants (single nucleotide polymorphisms (SNPs)) used as IVs are associated with exposures; 2) genetic variants are not associated with confounders; and 3) genetic variants influence the risk of outcomes only through interested exposures, not through other pathways [16] (Figure 1). The IVs (F statistic $>10)$ for all the exposures were sufficiently informative [17].

\section{Genetically predicted gut microbiota genera}

Genetic instruments of the abundance of 98 genera of gut microbiota at the level of genome-wide significance $\left(P<5 \times 10^{-8}\right)$ were obtained from available GWAS data of stool samples in humans [18]. As a 
result, independently significant SNPs were identified for 22 genera of the gut microbiota, but no significant genetic variants were found for the remaining 76 genera of the gut microbiota.

If an SNP was not available for an outcome, a highly correlated proxy SNP $\left(r^{2}>0.9\right)$ (https://Idlink.nci.nih.gov/) was used instead, if available. We checked the phenotypes of selected SNPs using comprehensive genotype-to-phenotype cross-references (GWAS Catalog[19]) and repeated the analysis with potentially pleiotropic SNPs excluded. We calculated SNP-specific $F$ statistics as a quotient of squared SNP-genus association and its variance [20].

\section{Genetically predictedgut microbial metabolites}

A transsynaptic, glutaminergic, excitotoxic mechanism (the so-called dying-forward hypothesis) has been proposed as a pathophysiological biomarker in ALS [21]. We therefore used 18 potential blood metabolites that might have causal effects on the development of ALS, including a group of gammaglutamyl amino acids [22]. The candidate metabolites were identified among 486 untargeted serum metabolites from Shin's study [23]. A total of 7824 adult individuals from 2 European cohorts were included in the GWAS analysis. Metabolomics data were acquired based on nontargeted mass spectrometry analysis of human fasting serum [23].

For each of the metabolites, we selected SNPs that showed an association at $\mathrm{P}<1 \times 10^{-5}$ as candidate IVs of the specific metabolite. Then, a clumping procedure was conducted with European $1000 \mathrm{G}$ as a reference panel to identify the independent variants, with a linkage disequilibrium threshold of $r^{2}<0.01$ in a 500-kb window.

\section{Genetically predicted ALS}

We drew on summary statistics from the largest and most recent GWAS of ALS [24] patients who were defined as having been diagnosed with probable or definite ALS according to the El Escorial criteria (Brooks, 1994) by a neurologist specializing in ALS. This GWAS of ALS involving 20,806 patients and 59,804 controls of European ancestry identified 10 independent genome-wide significant SNPs at the level of $\mathrm{P}<5 \times 10^{-8}[24]$.

\section{Statistical analysis}

For each direction of the potential relationship, we combined MR estimates using an inverse varianceweighted method (IVW) meta-analysis, which essentially translates to a weighted regression of SNP outcome effects on SNP exposure effects where the intercept is constrained to zero. The IV assumptions can be biased if instrument SNPs show horizontal pleiotropy, influencing the outcome through causal pathways other than exposure [16]. Therefore, other established MR methods, including weighted, weighted median mode, and MR Egger regression, were also applied to confirm the IVW results (number of SNPs $\geq 3$ ) because their estimates are known to be relatively robust to horizontal pleiotropy, although at the cost of reduced statistical power [25]. MR Egger regression allows the intercept to be freely 
estimated as an indicator of average pleiotropic bias. Effect estimates are reported in $\beta$ values when the outcome is continuous (i.e., the abundance of each genus of gut microbiota) and are converted to ORs when the outcome is dichotomous (i.e., ALS status).

To assess the robustness of significant results, we conducted further tests for horizontal pleiotropy using meta-analytic methods to detect heterogeneous outcomes, including leave-1-SNP-out analyses and the MR Egger intercept test of deviation from the null [26].

The analyses were performed with $\mathrm{R}$ version 3.1.1 ( $\mathrm{R}$ foundation) and Stata version 11.2 (Stata Corp, College Station, TX). All human research was approved by the relevant institutional review boards and conducted according to the Declaration of Helsinki. Ethical approval was obtained from relevant Research Ethics Committees and from the review boards of Peking University Third Hospital.

\section{Results}

\section{Effects of genetically predicted gut microbiota on ALS}

The resulting lists of instrument SNPs for each genus of gut microbiota are given in Table 1.

On the basis of 2 independent SNPs, OTU10032 unclassified Enterobacteriaceae was associated with a higher risk of ALS (per relative abundance: OR, 1.04; 95\% Cl, 1.01-1.07; $P=0.011$ ) (Figure 2, eFigure 1). Additionally, on the basis of 4 uncorrelated SNPs, unclassified Acidaminococcaceae was associated with a higher risk of ALS (per relative abundance: OR, 1.02; 95\% Cl, 1.01-1.04; $\mathrm{P}=0.009$ ) (Figure 2, eFigure 2). The independent SNPs for two genera with $r^{2}=0$ are listed in eTable 1 . Sensitivity analysis for the two candidate genera using the MR-Egger and weighted-median methods produced similar estimates, and no horizontal pleiotropy or outliers were observed (eTable 2-3).

Importantly, gamma-glutamyl amino acids showed potential deleterious effects on the risk of ALS. Gamma-glutamylphenylalanine (Gamma-Glu-Phe), a peptide in the gamma-glutamyl pathway, showed a significantly increased risk of ALS (genetically predicted per 1-standard deviation (SD) increase in the level of Gamma-Glu-Phe: OR, 1.96; 95\% Cl, 1.50-2.55; $P=0.012$ ) (Figure 3). In addition, two metabolites, 1-arachidonoyl-GPI and 3-methyl-2-oxobutyrate, were also estimated to be associated with a higher risk of ALS, with a genetically predicted per 1-SD increase in levels: $\mathrm{OR}, 1.64 ; 95 \% \mathrm{Cl}, 1.37-1.96 ; P=0.005$ for 1 arachidonoyl-GPI and $\mathrm{OR}, 2.78 ; 95 \% \mathrm{Cl}, 1.98-3.90 ; P=0.003$ for 3-methyl-2-oxobutyrate. The results also showed that a genetically predicted increase in the levels of 4-acetamidobutanoate may lower the risk of ALS (per 1-SD increase in levels: OR, $0.49 ; 95 \% \mathrm{Cl}, 0.36-0.66 ; P=0.020$ ). Sensitivity analysis for the metabolites using the MR-Egger and weighted-median methods produced similar estimates, and no horizontal pleiotropy or outliers were observed (eTable 4)

\section{Effects of genetically predicted ALS on gut microbiota}


On the basis of 2 independent SNPs, genetically predicted ALS was associated with an increase in the relative abundance of OTU4607_Sutterella (per 1-unit higher log odds: $\beta, 2.23 ; 95 \% \mathrm{Cl}, 1.27-$ $3.18 ; P=0.020$ ). The risk of ALS on each OTU4607_Sutterella-related SNP effect was estimated and is shown in eFigure 3. Similarly, on the basis of 2 independent SNPs, genetically predicted ALS was associated with an increase in the relative abundance of Lactobacillales_order (per 1-unit higher log odds: $\beta, 0.51 ; 95 \% \mathrm{Cl}, 0.09-0.94 ; P=0.019)$. Single Lactobacillales_ORDER-related SNP effect was estimated and is shown in eFigure 4. The estimated effects of ALS on the microbiota of each genus are listed in eTable 5. No horizontal pleiotropy or outliers were observed

\section{Discussion}

This study assessed the causal effects of potential microbiome modulators of human ALS and added intriguing evidence implicating some genera of the gut microbiome in modifying susceptibility to ALS. These genera attenuate ALS risk through gamma-glutamyl-related metabolite levels, supporting that a trans-synaptic, glutaminergic, excitotoxic mechanism could provide a pathogenic basis for ALS. These results may contribute to designing microbiome- and microbiome-dependent metabolite interventions in future ALS clinical trials. We further provide genetic evidence that the pathophysiology of ALS is associated with an altered relative abundance of the microbiota, strengthening the bidirectional relationship between the gut microbiota and ALS.

The gut microbiome is a source of these potentially disease-modifying bioactive metabolites and has recently been suggested to contribute to the pathogenesis of neurological disorders $[27,28]$. The family Enterobacteriaceae includes over 30 genera and 120 species of Enterobacteriaceae, but more than $95 \%$ of clinically significant strains fall into 10 genera and fewer than 25 species. All members of the Enterobacteriaceae family ferment glucose with acid production and nitrogen metabolism. Glutamine synthetases (GSs) are key enzymes of nitrogen metabolism, and their activity is modulated by nitrogen repression [29]. Acidaminococcaceae, an important glutamate-fermenting family of microbes, produces ammonia as the major end product through glutamate fermentation [30]. It is possible that alterations in the microbiomes of the two genera lead to changes in gamma-glutamyl-related metabolite levels. Circulating bioactive metabolites produced by the gut microbiome permeate the blood-brain barrier, after which they can play important roles in the pathogenesis of brain-related diseases [31].

Our study showed that higher ALS susceptibility was associated with a higher relative abundance of OTU4607_Sutterella and Lactobacillales_ORDER. In previous studies, gut dysbiosis, particularly reduced levels of butyrate-producing bacteria and higher E. coli and Enterobacteria abundance, was also found in ALS mice and ALS patients [6,32]. Furthermore, butyrate and short-chain fatty acids (SCFAs) produced by gut microbiota have been proposed as promising potential therapeutic agents affecting ALS progression $[33,34]$. However, unravelling the interplay between the gut microbiome and ALS is imperative, and more direct evidence and results are needed to clarify how the gut microbiota improves or aggravates ALS. 
There are several strengths in the present study, including the assessment of genera of gut microbiota and promising metabolites in relation to ALS, the use of data from the largest GWASs to date and bidirectional MR design. This design technique minimizes confounding by known and unknown factors and avoids reverse causation. In addition, consistent results from several sensitivity analyses, including the use of weighted mode, weighted median, and MR-Egger methods, indicate the robustness of our findings. Several limitations merit consideration. First, we used a limited number of gut microbiota and ALS SNPs as IVs; we cannot exclude that our findings might have been affected by weak instrument bias, although all genetic instruments were associated with exposure ( $F$-statistic $>10)$. Second, another potential source of bias in MR analyses is population stratification. We reduced this bias because the dataset for gut microbiota, metabolites and ALS was restricted to individuals of European ancestry. Replication with functionally relevant genetic prediction of gut microbiota is warranted given the substantial difference in gut microbiota composition among different populations. Finally, 16S rRNA gene sequencing only permits resolution from the genus to the phylum level rather than at a more specific level, resulting in biased results if some specific species contributed to ALS.

\section{Conclusion}

Our findings provide novel evidence supporting the bidirectional relationship between the gut microbiota and ALS and highlight that a transsynaptic, glutaminergic, excitotoxic mechanism could provide a pathogenic basis for ALS. These results may contribute to designing microbiome- and microbiomedependent metabolite interventions in future ALS clinical trials.

\section{Abbreviations}

ALS: amyotrophic lateral sclerosis

MR: Mendelian randomization

AM: Akkermansia muciniphila

IVs: instrumental variables

IVW: inverse variance-weighted method

SNPs: single nucleotide polymorphisms

GWASs: genome-wide association studies

OR: odds ratio

GS: glutamine synthetases

\section{Declarations}


Ethics approval and consent to participate

Ethical approval was obtained from relevant research ethics committees and from the review boards of Peking University Third Hospital.

\section{Availability of data and materials}

All data generated or analyzed during this study are included in this published article [and its additional file(s)].

\section{Competing interests}

The authors declare that they have no competing interests.

\section{Funding}

This study was supported by the National Natural Science Foundation of China $(81030019,81873784)$ and Beijing Key Laboratory of Biomarker and Translational Research in Neurodegenerative disorders.

\section{Authors' contributions}

D.F. and T.H. conceived and designed the study. L.Z., Z.Z. and G.Z. contributed to the acquisition and analysis of data. L.Z. wrote the manuscript. D.F. and T.H. reviewed and edited the manuscript. All authors read and approved the manuscript.

\section{Acknowledgments}

We are grateful that the summary data for all the gut microbiome, blood metabolite and AVS consortium studies are publicly available, and we thank all the investigators and participants who contributed to those studies.

\section{References}

1. Turner MR, Hardiman O, Benatar M, Brooks BR, Chio A, de Carvalho M, Ince PG, Lin C, Miller RG, Mitsumoto H, et al: Controversies and priorities in amyotrophic lateral sclerosis. Lancet Neurol 2013, 12:310-322.

2. Philips $\mathrm{T}$, Robberecht $\mathrm{W}$ : Neuroinflammation in amyotrophic lateral sclerosis: role of glial activation in motor neuron disease. Lancet Neurol 2011, 10:253-263.

3. Dupuis L, Pradat PF, Ludolph AC, Loeffler JP: Energy metabolism in amyotrophic lateral sclerosis. Lancet Neurol 2011, 10:75-82.

4. Trompette A, Gollwitzer ES, Yadava K, Sichelstiel AK, Sprenger N, Ngom-Bru C, Blanchard C, Junt T, Nicod LP, Harris NL, Marsland BJ: Gut microbiota metabolism of dietary fiber influences allergic airway disease and hematopoiesis. Nat Med 2014, 20:159-166. 
5. Sonnenburg JL, Bäckhed F: Diet-microbiota interactions as moderators of human metabolism. Nature 2016, 535:56-64.

6. Wu S, Yi J, Zhang YG, Zhou J, Sun J: Leaky intestine and impaired microbiome in an amyotrophic lateral sclerosis mouse model. Physiol Rep 2015, 3.

7. Zhang YG, Wu S, Yi J, Xia Y, Jin D, Zhou J, Sun J: Target Intestinal Microbiota to Alleviate Disease Progression in Amyotrophic Lateral Sclerosis. Clin Ther 2017, 39:322-336.

8. Fang X, Wang X, Yang S, Meng F, Wang X, Wei H, Chen T: Evaluation of the Microbial Diversity in Amyotrophic Lateral Sclerosis Using High-Throughput Sequencing. Front Microbio/ 2016, 7:1479.

9. Mazzini L, Mogna L, De Marchi F, Amoruso A, Pane M, Aloisio I, Cionci NB, Gaggia F, Lucenti A, Bersano E, et al: Potential Role of Gut Microbiota in ALS Pathogenesis and Possible Novel Therapeutic Strategies. J Clin Gastroenterol 2018, 52 Suppl 1, Proceedings from the 9th Probiotics, Prebiotics and New Foods, Nutraceuticals and Botanicals for Nutrition \& Human and Microbiota Health Meeting, held in Rome, Italy from September 10 to 12, 2017:S68-s70.

10. Brenner D, Hiergeist A, Adis C, Mayer B, Gessner A, Ludolph AC, Weishaupt JH: The fecal microbiome of ALS patients. Neurobiol Aging 2018, 61:132-137.

11. Blacher E, Bashiardes S, Shapiro H, Rothschild D, Mor U, Dori-Bachash M, Kleimeyer C, Moresi C, Harnik Y, Zur M, et al: Potential roles of gut microbiome and metabolites in modulating ALS in mice. Nature 2019, 572:474-480.

12. Lawlor DA, Harbord RM, Sterne JA, Timpson N, Davey Smith G: Mendelian randomization: using genes as instruments for making causal inferences in epidemiology. Stat Med 2008, 27:1133-1163.

13. Ding M, Huang T, Bergholdt HK, Nordestgaard BG, Ellervik C, Qi L: Dairy consumption, systolic blood pressure, and risk of hypertension: Mendelian randomization study. Bmj 2017, 356:j1000.

14. Geng T, Smith CE, Li C, Huang T: Childhood BMI and Adult Type 2 Diabetes, Coronary Artery Diseases, Chronic Kidney Disease, and Cardiometabolic Traits: A Mendelian Randomization Analysis. Diabetes Care 2018, 41:1089-1096.

15. Zhang L, Tang L, Huang T, Fan D: Life Course Adiposity and Amyotrophic Lateral Sclerosis: A Mendelian Randomization Study. Ann Neurol 2020, 87:434-441.

16. Bowden J, Davey Smith G, Burgess S: Mendelian randomization with invalid instruments: effect estimation and bias detection through Egger regression. Int J Epidemiol 2015, 44:512-525.

17. Pierce $B L$, Ahsan $H$, Vanderweele TJ: Power and instrument strength requirements for Mendelian randomization studies using multiple genetic variants. Int J Epidemiol 2011, 40:740-752.

18. Wang J, Thingholm LB, Skiecevičienè J, Rausch P, Kummen M, Hov JR, Degenhardt F, Heinsen FA, Rühlemann MC, Szymczak S, et al: Genome-wide association analysis identifies variation in vitamin D receptor and other host factors influencing the gut microbiota. Nat Genet 2016, 48:1396-1406.

19. Alkasir R, Li J, Li X, Jin M, Zhu B: Human gut microbiota: the links with dementia development. Protein Cell 2017, 8:90-102. 
20. Bowden J, Del Greco MF, Minelli C, Davey Smith G, Sheehan NA, Thompson JR: Assessing the suitability of summary data for two-sample Mendelian randomization analyses using MR-Egger regression: the role of the 12 statistic. Int J Epidemio/ 2016, 45:1961-1974.

21. Geevasinga N, Menon P, Özdinler PH, Kiernan MC, Vucic S: Pathophysiological and diagnostic implications of cortical dysfunction in ALS. Nature Reviews Neurology 2016, 12:651-661.

22. Yang $L, L v X, D u H$, Wu D, Wang M: Causal effects of serum metabolites on amyotrophic lateral sclerosis: A Mendelian randomization study. Prog Neuropsychopharmacol Biol Psychiatry 2020, 97:109771.

23. Shin SY, Fauman EB, Petersen AK, Krumsiek J, Santos R, Huang J, Arnold M, Erte I, Forgetta V, Yang TP, et al: An atlas of genetic influences on human blood metabolites. Nat Genet 2014, 46:543-550.

24. Nicolas A, Kenna KP, Renton AE, Ticozzi N, Faghri F, Chia R, Dominov JA, Kenna BJ, Nalls MA, Keagle P, et al: Genome-wide Analyses Identify KIF5A as a Novel ALS Gene. Neuron 2018, 97:12681283.e1266.

25. Hemani G, Bowden J, Davey Smith G: Evaluating the potential role of pleiotropy in Mendelian randomization studies. Hum Mol Genet 2018, 27:R195-r208.

26. Burgess S, Thompson SG: Interpreting findings from Mendelian randomization using the MR-Egger method. Eur J Epidemiol 2017, 32:377-389.

27. Sampson TR, Debelius JW, Thron T, Janssen S, Shastri GG, Ilhan ZE, Challis C, Schretter CE, Rocha S, Gradinaru V, et al: Gut Microbiota Regulate Motor Deficits and Neuroinflammation in a Model of Parkinson's Disease. Cell 2016, 167:1469-1480.e1412.

28. Sharon G, Cruz NJ, Kang DW, Gandal MJ, Wang B, Kim YM, Zink EM, Casey CP, Taylor BC, Lane CJ, et al: Human Gut Microbiota from Autism Spectrum Disorder Promote Behavioral Symptoms in Mice. Cell 2019, 177:1600-1618.e1617.

29. Tardito S, Oudin A, Ahmed SU, Fack F, Keunen O, Zheng L, Miletic H, Sakariassen P, Weinstock A, Wagner A, et al: Glutamine synthetase activity fuels nucleotide biosynthesis and supports growth of glutamine-restricted glioblastoma. Nat Cell Biol 2015, 17:1556-1568.

30. Gough EK, Stephens DA, Moodie EE, Prendergast AJ, Stoltzfus RJ, Humphrey JH, Manges AR: Linear growth faltering in infants is associated with Acidaminococcus sp. and community-level changes in the gut microbiota. Microbiome 2015, 3:24.

31. Kundu P, Blacher E, Elinav E, Pettersson S: Our Gut Microbiome: The Evolving Inner Self. Cel/ 2017, 171:1481-1493.

32. Mazzini L, Mogna L, De Marchi F, Amoruso A, Pane M, Aloisio I, Cionci NB, Gaggìa F, Lucenti A, Bersano E, et al: Potential Role of Gut Microbiota in ALS Pathogenesis and Possible Novel Therapeutic Strategies. J Clin Gastroenterol 2018, 52 Suppl 1, Proceedings from the 9th Probiotics, Prebiotics and New Foods, Nutraceuticals and Botanicals for Nutrition \& Human and Microbiota Health Meeting, held in Rome, Italy from September 10 to 12, 2017:S68-s70.

33. Erber AC, Cetin H, Berry D, Schernhammer ES: The role of gut microbiota, butyrate and proton pump inhibitors in amyotrophic lateral sclerosis: a systematic review. Int J Neurosci 2020, 130:727-735. 
34. Ghadge GD, Kay BK, Drigotas C, Roos RP: Single chain variable fragment antibodies directed against SOD1 ameliorate disease in mutant SOD1 transgenic mice. Neurobiol Dis 2019, 121:131-137.

\section{Tables}

Due to technical limitations, tables are only available as a download in the Supplemental Files section.

\section{Figures}

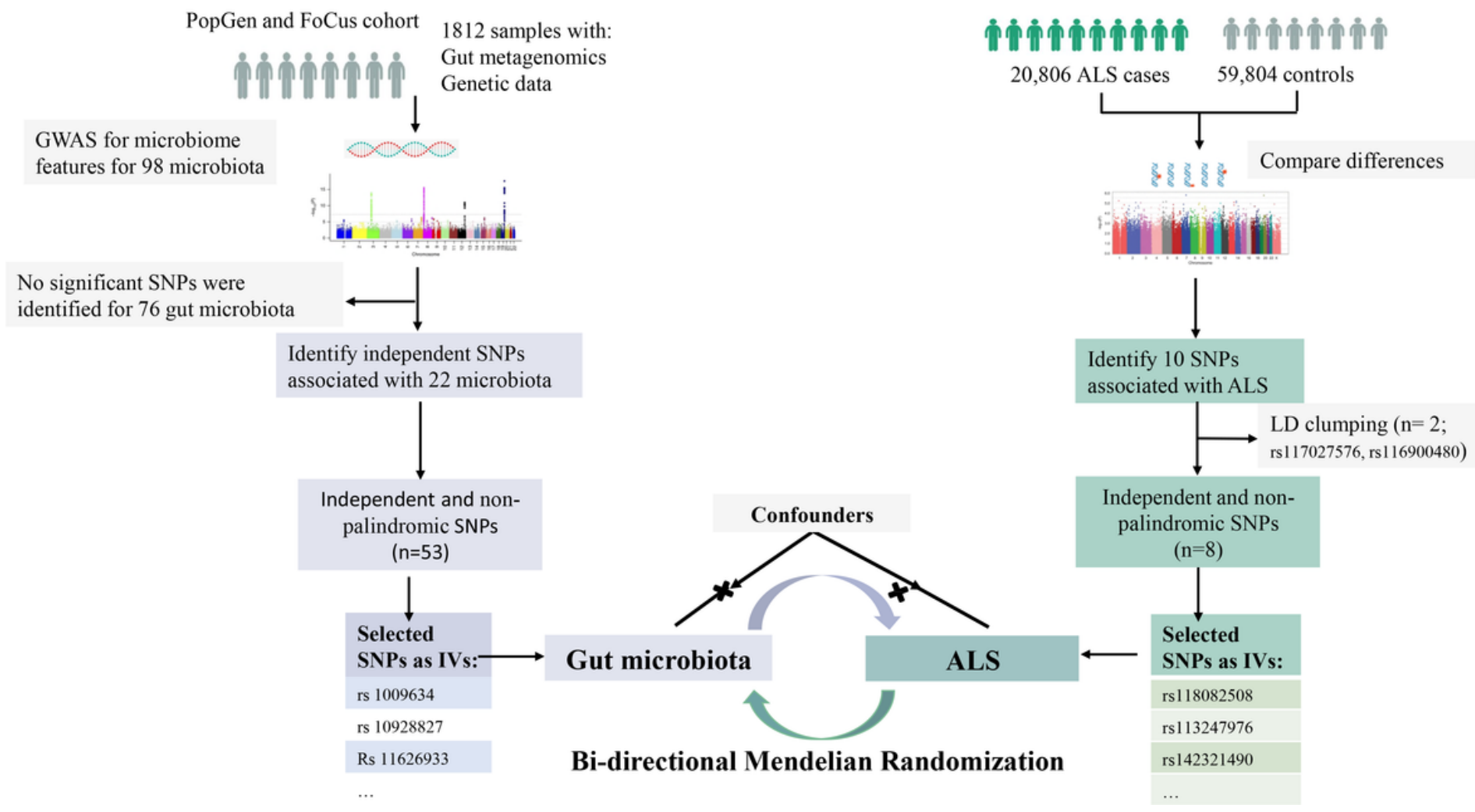

Figure 1

Schematic representation of the study. 


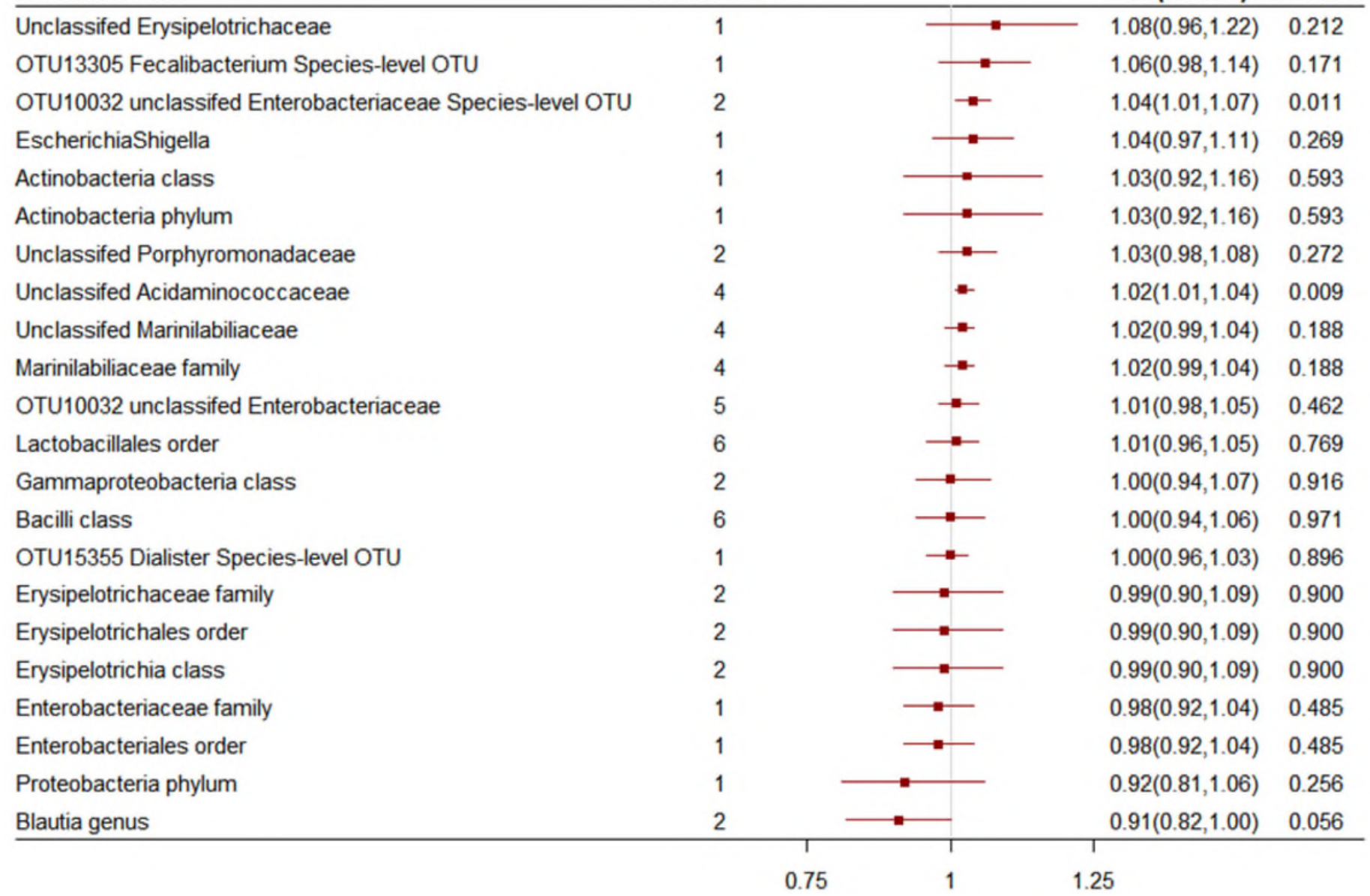

\section{Figure 2}

Odds ratio for association of genetically predicted gut microbiota with amyotrophic lateral sclerosis. OR: odds ratio; $\mathrm{Cl}$ : confidence internal. OR $(95 \% \mathrm{Cl})$ means risk of amyotrophic lateral sclerosis per 1-allele increase in single nucleotide polymorphisms related to greater abundance of gut microbiota.

\section{Genetic predicted}

Genetic predictors of gamma-Glu-Phe

\section{Blood metabolome}

Increased gamma-glutamyl amino acids

$$
\downarrow
$$

\section{Brain metabolites}

Increased levels of glutamate and glutamine<smiles>CCCCCCC</smiles>

\section{Motor neuron susceptibility}

Accelerated glutamate-mediated, excitotoxic mechanism

\begin{tabular}{llll} 
MR tests & OR $\mathbf{( 9 5 \%} \mathbf{~ C l )}$ & P-value \\
\hline Gamma-Glu-Phe & & & \\
IVW & $1.96(1.50-2.55)$ & $=0.012$ \\
Simple median & $2.54(1.76,3.67)$ & $=$ & 0.011 \\
Weighted median & $2.63(1.75,3.94)$ & $=0.017$ \\
MR Egger & $1.96(1.06,3.30)$ & - & 0.193 \\
\hline & & 0.84
\end{tabular}

OR $(95 \% \mathrm{CI})$ for amyotrophic lateral sclerosis 
Figure 3

Causal effect of microbiome-dependent metabolites on the risk of ALS. OR: odds ratio; Cl: confidence internal.

\section{Supplementary Files}

This is a list of supplementary files associated with this preprint. Click to download.

- Additionalfile1.xlsx

- e1.png

- e2.png

- e3.png

- e4.png

- graphicalabstract.png 